\title{
Laser Range and Bearing Finder for Autonomous Missions
}

\author{
Stephen R. Granade ${ }^{a}$ \\ ${ }^{a}$ Advanced Optical Systems, Inc., 6767 Old Madison Pike, Suite 410, Huntsville, AL 35806
}

\begin{abstract}
NASA has recently re-confirmed their interest in autonomous systems as an enabling technology for future missions. In order for autonomous missions to be possible, highly-capable relative sensor systems are needed to determine an object's distance, direction, and orientation. This is true whether the mission is autonomous in-space assembly, rendezvous and docking, or rover surface navigation. Advanced Optical Systems, Inc. has developed a wide-angle laser range and bearing finder (RBF) for autonomous space missions.

The laser RBF has a number of features that make it well-suited for autonomous missions. It has an operating range of $10 \mathrm{~m}$ to $5 \mathrm{~km}$, with a $5^{\circ}$ field of view. Its wide field of view removes the need for scanning systems such as gimbals, eliminating moving parts and making the sensor simpler and space qualification easier. Its range accuracy is $1 \%$ or better. It is designed to operate either as a stand-alone sensor or in tandem with a sensor that returns range, bearing, and orientation at close ranges, such as NASA's Advanced Video Guidance Sensor. We have assembled the initial prototype and are currently testing it. We will discuss the laser RBF's design and specifications.
\end{abstract}

Keywords: laser range and bearing finder, autonomous rendezvous and docking, space sensors, on-orbit sensors, advanced video guidance sensor

\section{INTRODUCTION}

The President's Vision for Space Exploration has set challenging goals for NASA and the US space community. In order to meet them, the Space Shuttle must be made safer, the International Space Station must be completed, and a crew exploration vehicle must be built. New missions call for unmanned craft to take samples and return them to Earth. At the same time, the nation no longer has a heavy-lift vehicle like the Saturn V, and is unlikely to develop one in the foreseeable future; because of this, final assembly of craft and support structures will need to be done on orbit. All of this leads to a dramatic increase in rendezvous and docking missions. The Hubble Space Telescope stands as an example of the cost of neglecting AR\&D: had it been designed with autonomous rendezvous in mind, the cost of servicing or deorbiting it would be significantly lower.

To support autonomous rendezvous and docking (AR\&D), sensors need to be developed and spacecraft designed with AR\&D in mind. Sensor development for AR\&D is ongoing in a number of areas. For example, NASA's Marshall Space Flight Center (MSFC) developed the Video Guidance Sensor (VGS) ${ }^{1}$ and, in conjunction with Advanced Optical Systems (AOS) and Orbital Sciences, the Advanced Video Guidance Sensor (AVGS) ${ }^{2,3}$; similarly, Optech and MD Robotics are developing the Rendezvous Laser Vision (RELAVIS). The VGS and AVGS work by illuminating several retro-reflector targets, imaging the reflected light, and processing the resultant pattern of light to determine range, bearing, and pose and provide six-degree-of-freedom (6DOF) information. Its working range is roughly $500 \mathrm{~m}$. RELAVIS uses a scanning LIDAR to track craft at typical ranges of $3.5 \mathrm{~km}$ in, and determines pose by matching the measured 3D image to models.

Under the auspices of a MSFC Small Business Innovative Research program, AOS has developed a wide-angle laser range and bearing finder (RBF) that falls between these two approaches. It is designed to provide range and bearing information from $5 \mathrm{~km}$ in to $10 \mathrm{~m}$. It can operate either as a stand-alone sensor or in tandem with a sensor that returns range, bearing, and orientation at close ranges, such as the AVGS. We are completing assembly of the initial prototype and will be testing it at the end of March. This paper discusses the laser rangefinder's design intent and resulting implementation. 


\section{DESIGN INTENT}

In developing the wide-angle laser RBF, AOS discussed mission requirements with potential end-users, both within NASA and at various commercial companies. To capture and document the requirements, we used Quality Function Deployment (QFD) methods ${ }^{5}$. QFD is a methodology for uncovering customer desires, both spoken and unspoken, and translating those desires into technical requirements. It addresses communication between customers and technical staff, and is excellent at relating qualitative desires to quantitative requirements. We also drew requirements from existing documents and presentations, such as the Interface Definition Document for ISS Visiting Vehicles and information gathered for a NASA 30-day study of autonomous rendezvous sensors.

Based on this work, we uncovered several things:

- There is an immediate need for a mid-range sensor for use in low-Earth orbit (LEO), to be a bridge between relative GPS/ground-based radar tracking and pose-estimating sensors. Sensors with a range beyond $5 \mathrm{~km}$ are not vital in LEO.

- A non-scanning system with a sufficient field of view is preferred over a scanning system, in order to make space qualification easier.

- In general, simpler methods are preferred over more complex ones.

- Leveraging existing flight heritage is preferred.

- For a number of potential missions, full $6 \mathrm{DOF}$ information is not necessary.

- Laser-based sensors in general hold much promise for AR\&D; laser rangefinders are of particular interest given the amount of research attention they have received in recent years.

This feedback led us to design a direct-detection laser RBF with a working range of $5 \mathrm{~km}$ in to $10 \mathrm{~m}$ and that would be compatible with AVGS. These two requirements in turn guided a number of design decisions.

For the laser RBF to be compatible with AVGS, it had to meet two requirements. One, it could not operate at the laser wavelengths used by AVGS, $808 \mathrm{~nm}$ and $845 \mathrm{~nm}$, in order to prevent crosstalk between the two sensors. Two, it had to be compatible with the AVGS targets. AVGS uses corner cube retro-reflectors, a common form of cooperative targets, with a piece of Schott BG36 absorptive filter glass over them. The filter glass allows AVGS to perform its foreground/background image subtraction: it transmits the AVGS foreground light at $845 \mathrm{~nm}$ and attenuates the background light at $808 \mathrm{~nm}$. To work with AVGS targets, then, the laser RBF must use a wavelength that is not $845 \mathrm{~nm}$ but that will not be attenuated by the BG36 filter glass. Figure 1 shows BG36 transmission as a function of wavelength.

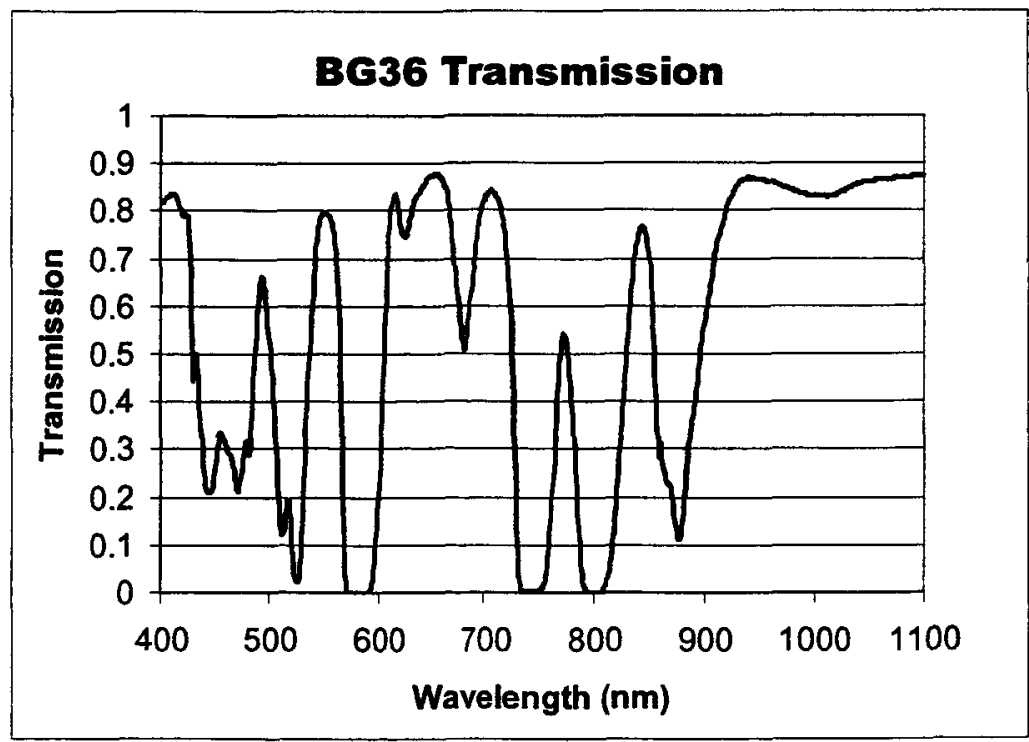

Figure 1. BG36 transmission as a function of wavelength. 
The wavelength dependence of BG36 filter glass helps drive the choice of laser. Another factor is the desire to have a near-eyesafe laser RBF: Longer wavelengths are more eye-safe. Our laser RBF employs multiple $2 \mathrm{~W}$ diode lasers operating at $940 \mathrm{~nm}$.

Laser rangefinders can be either monostatic or bistatic. In a monostatic laser RBF, the transmitter's optical axis is colinear with the receiver's; in a bistatic RBF, they are separate. Monostatic laser RBFs are more complex optically, and can result in crosstalk between the transmitter and receiver. However, they have a greater light return for a given ouput power, especially for cooperative targets such as corner cubes, since those targets reflect the light directly back at the source Figure? shows the reflected light in a monostatic and histatic system, and how much of the return light misses the receiver in the bistatic design. For our prototype laser RBF, we used a bistatic design for baseline testing, but also created a monostatic add-on to allow us to test both concepts.

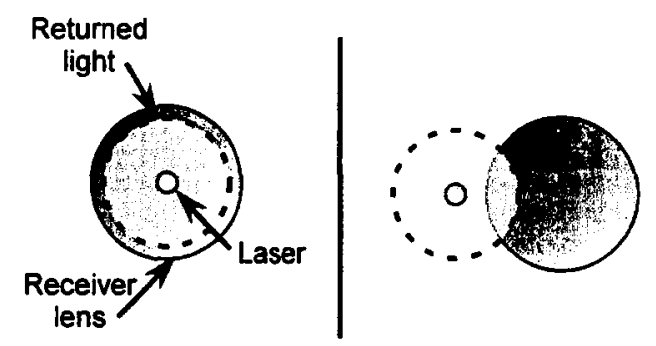

Figure 2. The light returned by corner cube targets for a monostatic (on the left) and bistatic (on the right) laser RBF. Note the light that misses the receiver in the bistatic design.

The choice of laser and laser power, when coupled with the desired range, help set both the transmitter and receiver field of view. The ranges that can be achieved with a laser RBF are governed by the laser radar range equation ${ }^{6}$, which specifies the amount of light a target returns to the receiver. It is given by

$$
P_{r}=\frac{K \Gamma D^{2} \eta_{t} \eta_{r}}{16 \pi \varphi^{2} r^{4}} P_{s},
$$

where $K$ is the profile of the transmitted beam, $\Gamma$ is the target's laser cross section, $D$ is the receiver aperture's diameter, $\eta_{t}$ is the transmitter's optical efficiency, $\eta_{r}$ is the receiver's optical efficiency, $\varphi$ is the beam width in radians, $r$ is the range from transmitter to target, and $P_{s}$ is the transmitter's laser power. $K$ accounts for the intensity pattern of the beam at the target's position? ${ }^{7}$. Figure 3 is a diagram corresponding to Equation 1.

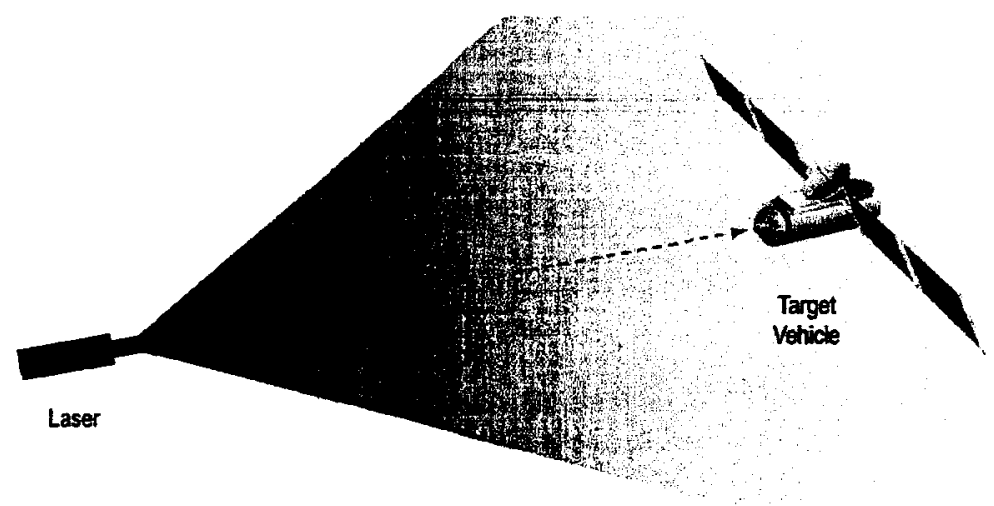




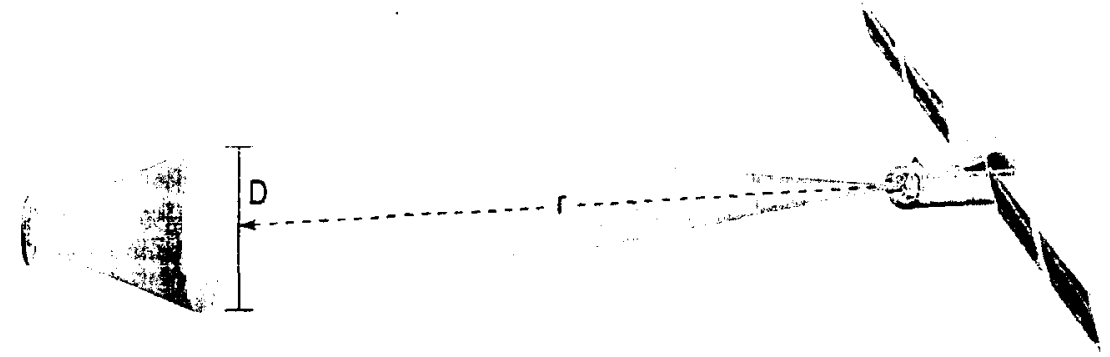

Figure 3. A diagram corresponding to the laser radar range equation. The top figure is of transmission; the bottom, of reception.

Using this equation, and for a fixed amount of output power, we can determine the amount of power received by the laser RBF for various fields of view. Figure 4 shows the amount of returned light at various fields of view. Based on initial calculations, we set our detection floor at $2 \times 10^{-6} \mathrm{~W}$, shown as a black line in Figure 4 . This led us to choose a field of view of $5^{\circ}$, as it was large without being unduly dim in return power.

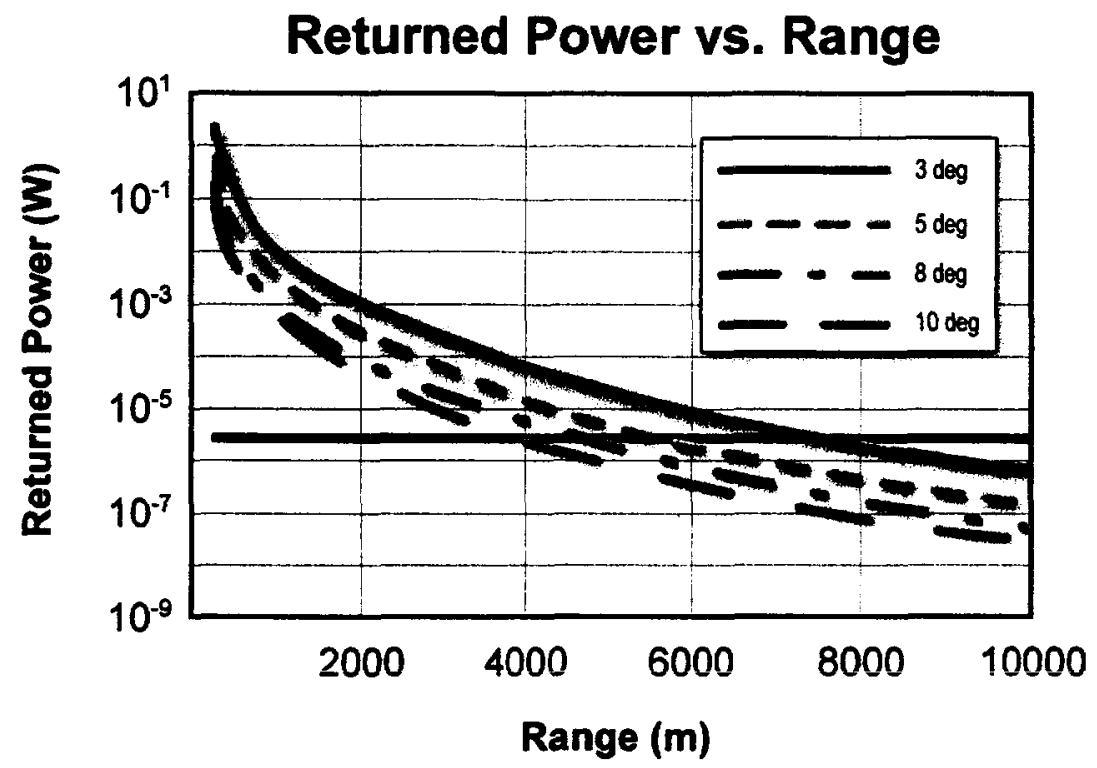

Figure 4. Amount of returned light at various fields of view, assuming that the transmitter and receiver have matched FOVs.

If the laser RBF is not to use a narrow scanned beam, then it must determine bearing by some other method. We chose to use a quadrant detector. A quad detector comprises four separate detectors arranged in quadrants. When a spot of light falls on the detector, each of the four quadrants produces a current proportional to the light's power. By comparing the different current levels of the two left quadrants to those of the two right ones, and by comparing the two top quadrants' currents to those of the bottom two, the spot's centroid can be calculated. The centroid in turn depends on the angle of the light returned from the targets, which gives the targets' bearing.

\section{IMPLEMENTATION}

Figure 5 shows a CAD model of our prototype laser RBF in bistatic mode. The laser RBF employs six diode lasers, firing three at a time; the other three are backups in case of laser failure. The prototype is larger than it needs to be, to provide easy access to all components and to allow air cooling of the boards. The space-qualified variant would be approximately half the size, with the ultimate size being driven by the size of the $10 \mathrm{~cm}$ receiver optics. 
Processing is done using a Xilinx Virtex-II XC2V3000 FPGA. Communications are handied by an Atmel 89LS52$16 \mathrm{AC}$ microprocessor. Both have radiation-hardened versions available. The lasers are driven by TI OP569 power amplifiers. Figure 6 is a block diagram of the electronics subsystem.

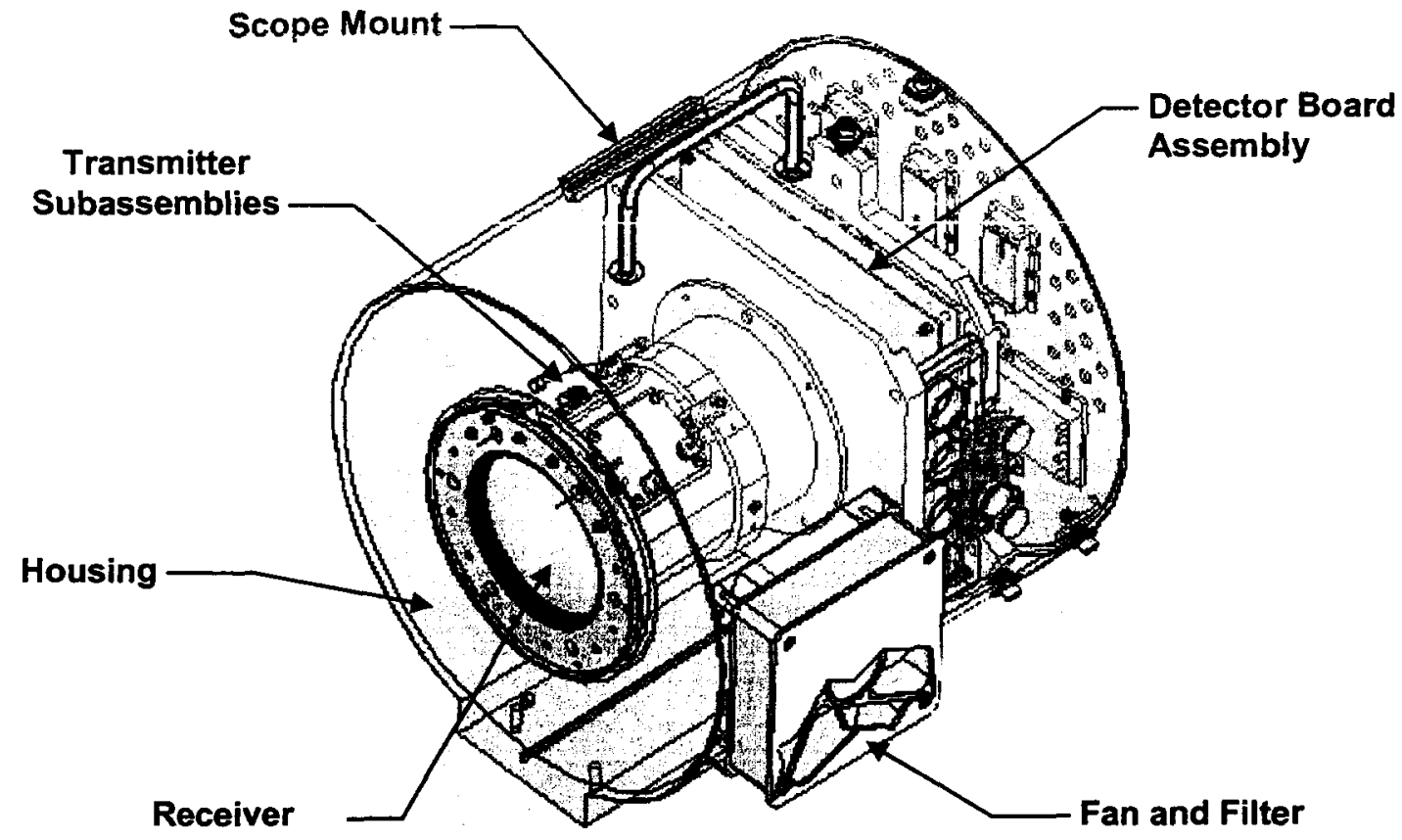

Figure 5. A CAD model of the prototype laser RBF in bistatic mode.

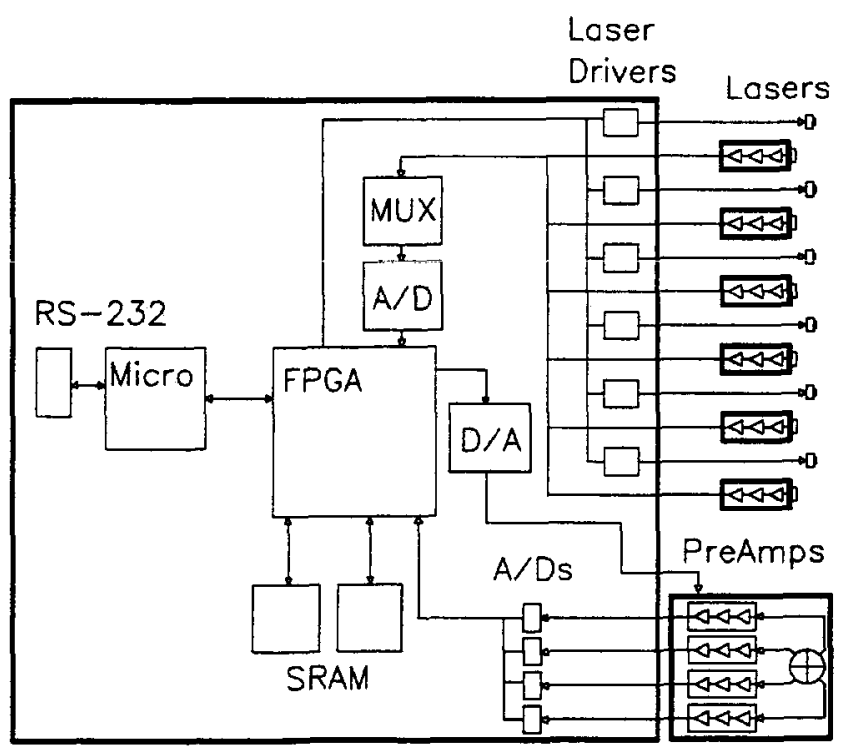

Figure 6. A block diagram of the electronics subsystem.

From the design considerations given in Section 2, we derived the specifications given in Table 1. One of them in particular bears more discussion: the signal processing scheme.

\begin{tabular}{|l|l|l|}
\hline & Parameter & Capability \\
\hline Range & $5 \mathrm{~km}$ & \\
\hline
\end{tabular}




\begin{tabular}{|c|c|}
\hline Range resolution & $1 \mathrm{~m}$ from $10 \mathrm{~m}$ to $100 \mathrm{~m} ; 1 \%$ of range thereafter \\
\hline Field of View & $5^{\circ}$ \\
\hline Bearing resolution & $0.1^{\circ}$ to $1 \mathrm{~km} ; 0.5^{\circ}$ to $5 \mathrm{~km}$ \\
\hline Output rate (range and bearing) & $5 \mathrm{~Hz}$ \\
\hline Output laser power & $6 \mathrm{~W}$ \\
\hline Mass & TK kg (estimated for final version) \\
\hline Volume (estimated for final version) & $\mathrm{TK} \mathrm{m}^{3}$ (estimated for final version) \\
\hline Average power & TK W (estimated for final version) \\
\hline Signal processing schientie & Contelatioñ maatching \\
\hline Pulse train length & $4.8 \mathrm{~ms}$ \\
\hline Individual pulse length & $200 \mu \mathrm{s}$ \\
\hline
\end{tabular}

Table 1. The laser range and bearing finder's specifications.

Laser rangefinders typically detect a return pulse and calculate its time of flight by establishing a signal threshold. To decrease false positives, techniques such as signal averaging or $m$-of- $n$ detection are used. We have chosen to use correlation matching: we correlate received light with transmitted light both to determine if a return signal exists and to calculate its time of flight.

In correlation matching, the output light is measured by a photodiode, providing our reference. The signals from the quad detector are then mathematically correlated with a waveform corresponding to the reference plus a time delay. The time delay is varied until the highest correlation value is found. The correlation value can be averaged or $m$-of- $n$ detection used to decrease false positives. While a signal threshold uses only the amplitude information in the received light, correlation uses more of the information in the received wave, such as the shape of its rising and falling edges. This increases our signal-to-noise ratio and allows us to pull signals out of the noise floor. The increase in signal-tonoise over a single pulse is approximately equal to

$$
S N R \cong \frac{T_{\text {Pulse }}}{T_{\text {Train }}},
$$

where $S N R$ is the increase in signal-to-noise ratio, $T_{\text {Pulse }}$ is the length of one pulse, and $T_{\text {Train }}$ is the length of one pulse train.

The use of correlation also allows us to take advantage of binary pulse encoding. The pulse trains are effectively a binary signal: if some pulses within the train are not sent, they are a 0 in the train. Transmitted pulses within the train are a 1. Different laser RBFs couid have different binary puises. Then, if several were operating in close proximity, they would not interfere with each other, as each laser RBF would only be correlating on its own laser pulse train.

Furthermore, this technique allows us to experiment with the kind of pulse encodings often used in radar, such as Barker $\operatorname{codes}^{8}$. Barker codes are specific codes that are mathematically optimal for producing the highest signal-to-noise ratio.

We are currently assembling the prototype laser RBF, and will begin testing it at the end of March.

\section{CONCLUSIONS}

Planned space missions are increasingly needing autonomous rendezvous and docking (AR\&D) capabilities, while current missions could use such capabilities to decrease their cost and risk. AR\&D sensor development is ongoing, though there is still more to be done. To support AR\&D missions, AOS has developed a wide-angle laser range and bearing finder that is designed to work either as a stand-alone system or in tandem with a sensor that returns 6DOF information, such as the Advanced Video Guidance Sensor. The laser RBF has a working range of $5 \mathrm{~km}$ in to $10 \mathrm{~m}$ with a range accuracy of $1 \%$ of the range. It provides bearing information with an accuracy of $0.5^{\circ}$, improving to $0.1^{\circ}$ at ranges of $1 \mathrm{~km}$ or less. We are finishing assembly of the initial prototype and will begin testing it at the end of March.

\section{ACKNOWLEDGMENTS}


This work was carried out as part of a Small Business Innovation Research program through NASA/Marshall Space Flight Center (MSFC).

\footnotetext{
'R.T. Howard, M.L. Book, and T.C. Bryan, "Video-Based Sensor for Tracking Three-Dimensional Targets," Proceedings of SPIE 4167 (2001).

${ }^{2}$ E. Tanner, S. Granade, and A. Whitehead, "Autonomous Rendezvous and Docking Sensor Suite," Proceedings of
} SPIE 5086 (2003).

${ }^{3}$ S. Van Winkle, "Advanced Video Guidance Sensor (AVGS) Project Summary," Proceedings of SPIE 5418 (2004).

${ }^{4}$ E. Martin, D. Maharaj, R. Richards, J. Tripp, J. Bolger, and D. King, "RELAVIS: The Development of a 4D Laser Vision System for Spacecraft Rendezvous and Docking Operations," Proceedings of SPIE 5418 (2004).

${ }^{5}$ Yoji Akao, Ed., Quality Function Deployment: Integrating Customer Requirements into Product Design, Productivity Press, New York, NY (1990).

"Gary W. Kamerman, "Laser Radar," in The Infrared and Electro-Optical Systems Handbook, Clifton Fox, Ed., vol. 6, p. 10, SPIE Optical Engineering Press, Bellingham, WA (1993).

${ }^{7}$ This is a specialized form of the laser radar range equation. Transmission losses due to the atmosphere have been neglected, since the proposed system will be space-based, and the assumption made that the sensor and target are moving slowly, if at all, with respect to each other.

${ }^{8}$ R. H. Barker, "Group Synchronizing of Binary Digital Systems," in Communications Theory, W. Jackson, ed., pp 273287, Academic Press, New York, NY (1953). 